\title{
Preanalytical Errors in Clinical Biochemistry Laboratory
}

\author{
Santosh Kumar', Archana Bharti², Sude Kumar Singh ${ }^{3}$ \\ ${ }^{1}$ Assistant Professor, Department of Biochemistry, DMCH, Darbhanga, Bihar Clinical, ${ }^{2}$ Clinical Biochemist, \\ Department of Biochemistry, Mahavir Cancer Sansthan, Patna, Bihar, ${ }^{3}$ Professor, HOD, Department of \\ Biochemistry, DMCH, Darbhanga, Bihar
}

\begin{abstract}
Introduction: In an era of evidence-based medicine, accuracy of test reports is mandatory. Automation of laboratories has drastically reduced the errors in the analytical phase of testing but errors in the preanalytical and postanalytical phases are largely responsible for the decrease in quality of clinical laboratory results. Objective of this study is to evaluate the leading causes of preanalytical errors in a clinical chemistry laboratory.

Method: The study was carried out in clinical biochemistry laboratory of Mahavir Cancer Sansthan, Patna. A prospective analysis of 3 months duration $1^{\text {st }}$ October to $31^{\text {st }}$ December has been carried out to evaluate errors in the preanalytical phase. samples from ward as well as out-patient samples were collected in the laboratory. Rejections of the samples as well as their causes for rejection were registered.

Results: 14,832 samples received during the data collection period. Out of these 639 samples were found unsuitable for further processing. This accounted for $4.18 \%$ of all samples collected. Reasons for the rejection were mentioned: hemolysis $-2.1 \%$; specimens with inappropriate or no patient i.d $-0.81 \%$; samples in inappropriate vacutainers $-0.62 \%$, inappropriate sample time $-0.31 \%$ (for fasting and postprandial plasma glucose, and lipid profile) and insufficient sample quantity $-0.33 \%$.

Conclusion: The overall percentage of rejection found is 4.18\%. Most Quality control programs at laboratory focuses only on the analytical phase, and the pre-analytical errors are neglected. Therefore, monitoring of pre and postanalytical phases for finding errors and corrective actions for the same are needed to achieve better test reporting.
\end{abstract}

Keywords: preanalytical errors, rejection, biochemistry laboratory,

\section{Introduction}

Accuracy of laboratory results is vital for the medical diagnosis and treatment monitoring of Patients. Despite advances in automation in diagnostic labs, there are still considerable error rates found. ${ }^{[1]}$ With continuous Improvement in sample collection, quality control

\section{Corresponding author:}

\section{Dr. Archana Bharti,}

MBBS, MD Biochemistry, Clinical Biochemist, Department of Biochemistry, Mahavir Cancer Sansthan, Patna, Bihar, India,

E-Mail id - archiemani82@gmail.com,

Mobile No- 8969544842 measures (internal and external), automation, and report dispatch, performance of laboratories has improved. But still we are not near to achieve $100 \%$ accuracy and precision. The pre-and post-analytical phases of the process account for major errors to be corrected.

According to the ISO 15189: 2012 standard for laboratory accreditation the preanalytical phase that comprises of all the procedures and processes in the initial phase of the Total Testing Process needs to be evaluated, monitored and improved. Pre analytical phase is the most vulnerable part of the Total Testing Process.

The testing process in a clinical biochemistry laboratory starts with test requisition form filled by the physician followed by the sample given to the 
laboratory (preanalytical phase), analysis of the samples (analytical phase), interpretation of report and dispatch of report to the patient or to the physician (postanalytical phase). Laboratory errors may occur at any part of these three phases and it is not exclusive in a particular phase. Errors at any point will alter the test report and can cause inappropriate clinical decisions, delayed or wrong diagnoses and increases the demand of resources in the form of re-sampling or retesting. [2] in the era of automation, it has been found that Pre- and postanalytical phases have been recognized as a larger source of laboratory errors; pre-analytical $(46 \pm 68 \%)$ and post analytical $(18 \pm 47 \%)$, While only $7 \pm 13 \%$ of errors occur during the analytical phase. ${ }^{[2]}$ In the preanalytical phase, many professionals as physicians, specialists of laboratory medicine, nurses, laboratory technicians and phlebotomists are involved and is the hardest to regulate and monitor. The most common pre-analytical errors include inappropriateness of test order, patient identification error, timing errors in sampling and preparation, hemolytic and lipemic blood samples, inappropriate transport, and inadequate and inappropriate sample collection tubes.

The objective of the present study is to raise awareness of the importance of quality control measures for the pre-analytical phases.

\section{Materials and Method}

A prospective Study On "Pre-Analytical Errors in a Clinical Biochemistry Laboratory" was conducted at Dept. of Biochemistry, Mahavir Cancer Sansthan.

A total of 14,832 samples were analyzed in the clinical biochemistry laboratory during the period of 3 months from October 2019 to December 2019.

Laboratory personnel were asked to make entries of rejected samples and their cause. Every day entries were monitored.

\section{Errors monitored are}

1. Incorrect sample identification

2. inappropriate vacutainers

3. inappropriate test identification
4. Inadequate Sample: sample received is not sufficient for testing,

5. Wrong timing for Collection - lipid profile, fasting/pp plasma glucose

6. Haemolysed Sample: Presence of pink to red tinge in serum or

Plasma.

OPD samples are collected at centralized collection center by the lab personal between 9 AM-4 PM in colour coded vacutainers. Patient id and the test to be done are mentioned and barcode is leveled, are placed in a separate rack and sent to the lab at regular one-hour interval. Inpatient blood samples are collected by the nursing staff in the respective wards and delivered to the lab by paramedical staff or patient relatives. Once samples are received in the lab, they are centrifuged to obtain serum/ plasma for test analysis. Samples with inappropriate or no patient id, tests to be done not mentioned, inappropriate colour coding, insufficient or no sample are rejected and mentioned. After centrifugation hemolysed samples are rejected and noted. Sample time errors are found after test reporting and patient history at the time of report dispatch. Fully Automated machines are used to analyze the samples like integrated system of Automated Biochemistry Analyzer, Electrolyte Analyzers and Automated Immunoassay Analyzer (Abbott Architect Ci4100). Analytical phase is also ensured with the calibrations and quality control runs (internal quality control and external quality controls).

\section{RESULTS}

Out of 14832 samples, $4.18 \%$ pre-analytical errors were observed (639 samples). 122 samples were wrongly identified $(0.81 \%), 92$ samples were taken in inappropriate collection vial $(0.62 \%) .50$ samples were inadequate $(0.33 \%), 45$ samples were collected in the wrong time $(0.31 \%), 316$ samples were hemolysed $(2.1 \%)$ and 50 sample were with insufficient sample quantity $(0.33 \%)$.

All these samples were rejected for the testing and all these patients were advised for repeat fresh sample. 


\section{preanalytical errors}

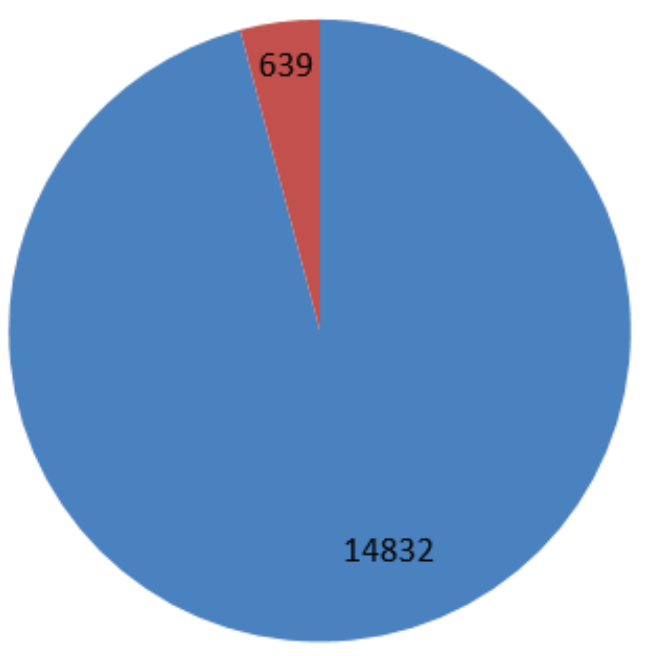

- Total Sample

- Preanalytical Error (4.18\%)

\section{pattern of preanalytical errors}

n hemolysed sample

- identification error

wrong vacutainers

n insufficiant sample quantities $\mathbf{m}$ wrong time sampling

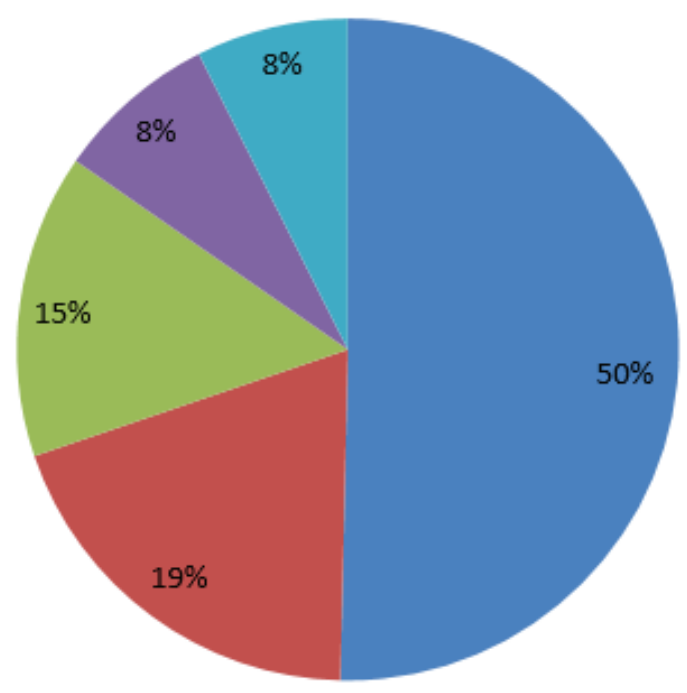




\section{Discussion}

In this study we have discussed about the errors that hampers accuracy in test reporting. Errors encountered related to testing phases can be classified as

\section{Pre-analytical phase}

1. error in Test Requisition Form

2. inappropriate sample identification

3. Incorrect vacutainers

4. Sample from IV line

5. Delay in sample transportation

6. samples Insufficient for tests

7. Sample mix-ups

8. Broken tube in centrifuge

9. inappropriate timing for sample Collection

10. Haemolysed / Lipemic / Icteric Sample

11. Software errors

Analytical phase

1. Instrument calibration

2. Specimen mix-up

3. Inadequate specimen

4. Presence of interfering substances

5. Wrong analytical method

Post-analytical phase

1Transcriptional error

2. Report delayed

3. Report not legible

4. Previous values are not available for comparison

5. error in patient identification

Currently, many studies are focusing on the importance of pre-analytical phase to reach accuracy in lab results. An American Pathologist program conducted a study with 660 laboratories enrolled and $4.8 \%$ error rate was found from outpatient department only. [3] Another study conducted by The College of American Pathologists, with 120 labs, found misidentification of samples as the commonest laboratory error. Another more extensive study by Danish on laboratory errors found $81 \%$ pre-analytical errors $(82.6 \%$ human errors and $4.3 \%$ technical errors) and only $10 \%$ analytical errors. ${ }^{[4]}$

In our study, we observed $4.18 \%$ of different types of pre-analytical errors in clinical biochemistry laboratory at our institute. The most common error was found to be hemolysis, which accounted for $2.1 \%$ of total sample rejection which is similar to many other studies. ${ }^{[5]}$ It was also noted that samples from indoor patients were more prone to hemolysis than the OPD samples. The various causes for hemolysis found to be Cleansing the venipuncture site with alcohol and not allowing the site to dry appropriately $(30 \mathrm{sec})$, transferring the sample into a tube by pushing down on the syringe plunger to force blood into a tube, vigorous mixing of the samples and not allowing the serum specimen to clot for the recommended time can result in fibrin formation in the serum. ${ }^{[6]}$ Hemolysis has a profound influence on various parameters. Parameters like Potassium, Alanine Transaminase (ALT), Creatinine, Creatine Kinase (CK) are overestimated,

Whereas parameters like albumin, alkaline phosphatase (ALP), chloride, gamma glutamyl transferase (GGT), glucose, bilirubin and sodium are underestimated.

In our study, we found $0.82 \%$ Incorrectly identified samples for the rejection due to heavy work load. It is very important to identify a patient accurately. Patient identification is the critical first step in blood collection. This has the worst clinical outcomes. Drawing blood from the wrong person, or labeling the correct patient's sample with a different patient's label can certainly contribute to laboratory error. When identifying the patient, their full name, age, address, hospital identification number must be confirmed. Hospital identification band with these records can be given to the inpatients.

The next common error that we found in our study is inadequate sample, causing $0.33 \%$ sample rejection. The cause for this can be lack of the knowledge about 
the testing volume, not reading the number of tests requested in test requisition form, difficulty in sampling in pediatric cases, debilitating diseases, and shortage of manpower.

\section{Conclusion}

It is crucial to get accurate and precise test reports for the patient care. It is very important to monitor all the steps involved in the sample collection, processing and analysis of samples, and the dispatch of the reports. Our study recommends the training of phlebotomy staff on various aspects as an important quality control measure in the preanalytical phase.

Ethical Clearance: Taken

Source of Funding: Self

Conflict of Interest: None

\section{References}

1. Baron JM, Mermel CH, Lewandrwski KB, Dighe AS. Detection of preanalytical laboratory testing errors using statistically guided protocol. Am J Clin Pathol. 2012; 138:406-413.
2. Cornes MP, Atherton J, Pourmaharam G, Borthwick $\mathrm{H}$, Kyle B, West J, et al. Monitoring and reporting of pre-analytical errors in laboratory medicine: the UK situation. Ann Clin Biochem. 2016; 53:279284.

3. Valenstein P, Meler F. Outpateint order accuracy. A College of American Pathologists Q-probes study of requisition order entry accuracy in 660 institutions. Arch Pathol Lab Med. 1999; 123:11451150 .

4. Lippi G, Salvango GL, Montaganna M, Brocco $\mathrm{G}$, Guidi GC. Influence of hemolysis on routine clinical chemical testing. Clin Chem Lab Med. 2006; 44:311-316.

5. Koseoglu M, Hur A, Atay A, Cuhadar S. Effects of hemolysis interference on routine biochemistry parameters. Biochem Med. 2011; 21:79-85.

6. Calmarza P, Cordero J. Lipemia Interferences in routine clinical biochemical tests. Biochem Med. 2011; 21:160-166. 